\title{
A preliminary study of THC-LSD interactions on rabbit EEG and behavior
}

\author{
PARTHENA MARTIN \\ Department of Psychology, Virginia State University, Petersburg, Virginia 23803 \\ and \\ PAUL CONSROE \\ Department of Pharmacology and Toxicology, College of Pharmacy, University of Arizona \\ Tucson, Arizona 85721
}

\begin{abstract}
Quantified cortical and hippocampal electroencephalograms (EEG) and corresponding behaviors were recorded from rabbits who were given either acute or subacute (12-day) delta-9tetrahydrocannabinol (THC; .5 mg/kg, iv) followed by acute lysergic acid diethylamide (LSD; $50 \mu \mathrm{g} / \mathrm{kg}$, iv). The results indicated that acute THC does not block the stimulant effects of LSD (increases in standing and activity). However, subacute THC produced tolerance to the depressant cortical EEG (increase in delta) and behavioral (sprawling) activity of the cannabinoid and blocked some excitatory effects of LSD.
\end{abstract}

During recent years, there has been much selfexperimentation with psychoactive drugs, especially with two or more drugs being used simultaneously. For example, data recently compiled by the National Institute on Drug Abuse (Dupont, 1976) show that $39 \%$ of the patients admitted for treatment of problems due to use of hallucinogens such as lysergic acid diethylamide (LSD) also have secondary drug problems due to use of marijuana. However, only a few studies have examined the resultant pharmacological interaction of hallucinogens and marijuana or its major psychoactive ingredient, delta-9tetrahydrocannabinol (THC). In rats (Pryor, 1976; Pryor, Husain, Mitoma, \& Braude, 1967), acute LSD was shown to augment some depressant effects of acute THC, but attenuation of these LSD effects resulted when the animals were made tolerant to the effects of the cannabinoid (i.e., with 6-day subacute THC administration). Moreover, additional effects in humans (Isbell \& Jasinski, 1969) and other animals (Silva, Terassa, Carlini, Clausser, \& Korke, 1968) showing tolerance to chronic LSD did not exhibit cross-tolerance to THC.

The present investigation was carried out to examine further the potential pharmacological interactions between LSD and THC. Utilizing quantified electroencephalographic (EEG) measurements and behavioral observation in rabbits, the present study examined the interactive effects of LSD following acute and 12-day subacute administration of THC.

This research was supported by Grant DA01448 and by supplies of THC and LSD from the National Institute on Drug Abuse.

\section{METHOD}

\section{Animals}

Two female and two male New Zealand white rabbits, weighing 2.3 to $3.4 \mathrm{~kg}$, were used. Each subject was housed individually in a room maintained at constant temperature $\left(25^{\circ} \pm 2^{\circ} \mathrm{C}\right)$ and under controlled lighting (12-h light-dark cycle). Animals were allowed ad-lib access to food and water except during testing.

\section{Electrode and Catheter Implants}

Electrodes were stereotaxically implanted in the motor cortex and the right dorsal hippocampus under chlorpromazinepentobarbital anesthesia. All electrodes were connected to an amphenol connector anchored to the cranium with stainless steel screws and dental acrylic cement. At the same time, a catheter was implanted in the right jugular vein, which permitted drug infusion from outside the observation chamber without disturbing the rabbit's behavior. All animals were allowed 7-10 days to recover from surgery before testing.

\section{Drugs}

LSD tartrate was prepared in a solution of isotonic saline, while THC was incorporated into a $10 \%$ polysorbate (Tween) 81-saline emulsion. Drugs and vehicles were given intravenously (iv), and doses of THC $(.5 \mathrm{mg} / \mathrm{kg})$ and LSD $(50 \mu \mathrm{g} / \mathrm{kg})$ were calculated as the active moiety. These doses were employed in the present study since data from other studies (Consroe, Jones, \& Chin, 1975; Consroe, Jones, \& Martin, 1977) have shown that these doses (given singly) produce consistent behavioral and EEG patterns in rabbits without signs of behavioral toxicity.

\section{Apparatus}

Freely moving animals were tested in a sound-resistant test chamber measuring $82 \mathrm{~cm}$ square $\times 70 \mathrm{~cm}$ high. A one-way vision window permitted continuous observation of animal behavior. Shielded EEG cable connected the electrodes from the rabbit to a Grass Model 7 B polygraph, where an EEG frequency analysis system (described in Martin \& Consroe, 1978) removed highvoltage movement artifacts, then separated the EEG into delta $(.5-4.0 \mathrm{~Hz})$, theta $(4.0-8.0 \mathrm{~Hz})$, alpha $(8.0-13.0 \mathrm{~Hz})$, and beta $(>13.0 \mathrm{~Hz})$ frequency bands. The area under successive EEG waves 
for each band was integrated, and the digital cumulative summation was visually displayed. Additionally, a manually operated 10-channel event recorder was used to quantify the corresponding behavior of the rabbits.

\section{Dependent Variables Measured}

The bipolar electrical activity between the left and right motor cortex and from the dorsal hippocampus was assessed qualitatively; additionally, the energy spectrogram, calculated as percent delta, theta, alpha, and beta EEG frequencies, was also recorded. In addition to EEG frequencies, the frequency and duration of the following behaviors were measured: (1) standing-weight of the subject supported on the tarsals and front legs extended vertically, with the subject up on all 4 legs; (2) sprawling-weight of the subject distributed along the ventral body surfaces, with two or more legs extended laterally (splayed) and/or head touching chamber floor; and (3) activity-measured as the combined score of the following behaviors: (a) grooming-licking or scratching directed toward the subject's body, (b) rearing-standing up, with the front paws off the floor of the chamber, (c) locomotionmovement of a distance equal to or greater than one-half the body length of the subject, and (d) exploring-sniffing at the chamber or at the object in the chamber, with extended head and vibrissae movements.

\section{Procedure}

After recovering from surgery, rabbits were given three weekly 40-min exposures to the testing conditions with control vehicle treatment so that they became adapted to the test chamber and to the injection procedure.

Following adaptation, on Day 1 of the experiment, each rabbit was injected with $10 \%$ Tween 81-saline at the beginning of the first 20-min period and isotonic saline at the beginning of the second 20-min period, thus providing the vehicle-control data. On Days 2 through 12 of the experiment, each rabbit received an injection of THC $(.5 \mathrm{mg} / \mathrm{kg})$ with saline injected $20 \mathrm{~min}$ after the THC on the first and last days of the THC injections, forming the acute THC and subacute THC treatment groups. On Day 13 of the experiment, each rabbit was given an injection of THC $(.5 \mathrm{mg} / \mathrm{kg})$ followed $20 \mathrm{~min}$ later by an injection of LSD $(50 \mu \mathrm{g} /$ kg), forming the subacute THC-LSD interaction treatment.

Two weeks after the above drug-interaction treatment, during which time no drugs were administered, animals were tested with LSD alone, that is, with Tween 81-saline administered at the beginning of the first 20 -min period and LSD $(50 \mu \mathrm{g} / \mathrm{kg})$ administered at the beginning of the second 20-min period. After another 2 weeks of no drug treatment, rabbits were injected with THC $(.5 \mathrm{mg} / \mathrm{kg})$ followed $20 \mathrm{~min}$ later by $\operatorname{LSD}(50 \mu \mathrm{g} / \mathrm{kg})$.

Data from the second 20-min testing period were analyzed using a repeated measure analysis of variance (ANOVA). A Tukey HSD test was used for post hoc comparisons.

\section{RESULTS}

While none of the treatments produced any significant changes from control in the hippocampal EEG, there were profound effects on cortical EEG patterns. As shown in Figure 1, LSD given alone (panel A) elicited cortical EEG desynchronization, whereas acute THC (panel B) caused EEG synchronization. Following acute THC, the LSD-induced EEG desynchronization was greatly reduced (panel B). Moreover, subacute THC evinced EEG signs of tolerance and also more completely blocked the activating effects of LSD on the EEG (panel C).

The mean and standard errors of each treatment group for cortical delta, theta, alpha, and beta fre-
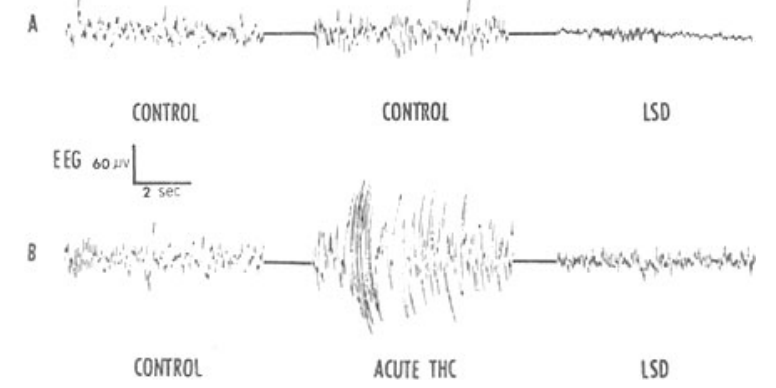

CONTROL
SUBACUTE THC

Figure 1. Representative cortical EEG tracings of treatment effects in rabbits.

quencies are shown in Table 1. There was a significant main effect among treatment groups for theta $[F(5,15)=3.845, p<.05]$ and trends toward treatment differences for delta $[\mathrm{F}(5,15)=2.496, .05<\mathrm{p}$ $<.10]$ and beta $[F(5,15)=2.67, .05<\mathrm{p}<.10]$ EEG frequencies. Post hoc comparison of treatment revealed that LSD given alone produced a differential decrease $(p<.05)$ in theta compared with the acute THC group. Additionally, there was a reduction in beta following subacute THC-LSD administration compared with the LSD-alone treatment $(p<.05)$. It could be argued that since THC has been found to persist in the body fat and spleen of rabbits for as long as 3 days (Agurell, Milsson, Ohlsson, \& Sandberg, 1970), the LSD-alone group should have consisted of animals not previously tested with THC. However, since the data from the LSD-alone group are quite consistent with data from other studies looking at the effects of LSD alone, and since a clear difference was found between the LSD-alone group and the LSD-THC group, the 2week period of time between testing seems to have been adequate to control for any persistent effect of THC.

The means and standard errors of treatment groups for standing, activity, and sprawling behaviors are also shown in Table 1 . The ANOVA revealed differences between groups for standing $[F(5,15)=18.29$, $\mathrm{p}<.001]$ and activity $[\mathrm{F}(5,15)=6.563, \mathrm{p}<.01]$. Moreover, only acute THC produced sprawling, and this unique behavior disappeared completely by the last day of THC administration (i.e., in the subacute THC group). LSD given alone produced an increase in the mean duration of standing compared with acute THC alone $(p<.05)$, subacute THC alone $(p<.05)$, and vehicle control $(p<.05)$. LSD given after both acute THC and subacute THC also resulted in an increase in standing behavior compared 
Table 1

Mean Percent of Cortical EEG Frequencies and Behaviors for Each Treatment Group

\begin{tabular}{|c|c|c|c|c|c|c|c|c|c|c|c|c|c|c|}
\hline \multirow[b]{3}{*}{ Treatment Group } & \multicolumn{8}{|c|}{ Motor Cortex } & \multicolumn{6}{|c|}{ Behavior } \\
\hline & \multicolumn{2}{|c|}{ Delta } & \multicolumn{2}{|c|}{ Theta } & \multicolumn{2}{|c|}{ Alpha } & \multicolumn{2}{|c|}{ Beta } & \multicolumn{2}{|c|}{ Stand } & \multicolumn{2}{|c|}{ Activity } & \multicolumn{2}{|c|}{ Sprawl } \\
\hline & Mean & SEM & Mean & SEM & Mean & SEM & Mean & SEM & Mean & SEM & Mean & SEM & Mean & SEM \\
\hline Vehicle-Vehicle & 43.7 & 3.9 & 25.8 & 2.8 & 17.4 & 2.1 & 12.4 & 2.0 & 14.1 & $1.4 * \dagger$ & 5.5 & $2.8 \dagger$ & .0 & .0 \\
\hline Acute THC-Vehicle & 33.7 & 2.3 & 38.5 & $7.2 *$ & 15.8 & 4.1 & 11.7 & 4.3 & 2.5 & $2.5 * \dagger$ & .8 & $.4 \dagger$ & 21.5 & $20.4 * \dagger$ \\
\hline Subacu te THC-Vehicle & 41.0 & 1.7 & 28.2 & 1.1 & 18.7 & 1.5 & 11.5 & 4.0 & 3.7 & $1.4 * \dagger$ & 3.4 & $1.8 \dagger$ & .0 & .0 \\
\hline Vehicle-LSD & 42.7 & 4.6 & 15.2 & 5.4 & 16.0 & 6.0 & 25.6 & 4.1 & 85.2 & 3.2 & 10.8 & 4.0 & .0 & .0 \\
\hline Acute THC-LSD & 28.2 & 6.5 & 18.9 & 2.4 & 13.7 & .9 & 14.6 & 1.3 & 67.0 & 12.0 & 20.7 & 10.2 & .0 & .0 \\
\hline Subacu te THC-LSD & 45.7 & 2.9 & 30.8 & 4.7 & 18.8 & 1.2 & 7.0 & $4.0^{*}$ & 56.9 & 16.1 & .7 & $.5 \dagger$ & .0 & .0 \\
\hline
\end{tabular}

*Comparison with vehicle-LSD treatment $(p<.05)$. †Comparison with acute THC-LSD treatment $(p<.05)$.

with acute THC alone $(p<.05)$, subacute THC alone $(p<.05)$, and vehicle control $(p<.05)$. In addition, LSD administered following acute THC produced an increase in activity compared with vehicle control $(p<.05)$, acute THC alone $(p<.05)$, subacute THC alone $(\mathrm{p}<.05)$, and subacute THC$\operatorname{LSD}(\mathrm{P}<.05)$.

\section{DISCUSSION}

The results of the present study are in general agreement with previously published data showing that EEG and/or behavioral stimulation occur with acute LSD (Consroe et al., 1977; Isbell \& Jasinski, 1969), while those of depression predominate with acute THC (Consroe et al., 1975; Pryor et al., 1976; Silva et al., 1968) and that tolerance develops to the depressant effects of the cannabinoid upon continual administration (Dewey, Martin, \& Harris, 1976; Pryor et al., 1976; Silva et al., 1968). Although THC has been reported to disrupt hippocampal theta rhythm (Fujimori \& Himwich, 1973; Lipparini, de Carlos, \& Longo, 1969), Martin and Consroe (1978) found that THC has effects primarily on the voltage and regularity of hippocampal EEGs rather than on the frequency, so it is not surprising that no effect of THC on hippocampal EEG frequencies was found in the current experiment.

Although only single doses of THC and LSD were used in the present study, these results also indicate that at these doses, acute THC does not block the stimulant effects of LSD (increases in standing and activity), while subacute THC does attenuate some excitant EEG effects of the hallucinogen. Pryor et al. (1976) found that the depressant effects of acute THC augment the acute depressant effects of LSD on photocell activity, heart rate, blood pressure, conditioned avoidance, and rotorod performance in rats. Additionally, he found that tolerance to subacute THC extends to the interaction of THC and LSD, causing an attenuation of the predominant depressant effects of their acute combination. It is difficult to make detailed comparisons between these two studies in view of the divergent experimental species and methods employed-notably, the dependent variables that manifested primarily stimulant or depressant responses to LSD given singly. Nevertheless, both studies clearly indicate that there is a pharmacological interaction between LSD and THC and that the nature of this interaction depends on the individual effects of each drug and whether THC is given on acute or subacute basis. The interaction may involve something other than a direct interaction at the receptor level. Studies in rats (Silva et al., 1968) and humans (Isbell \& Jasinski, 1969) showing a lack of cross-tolerance between LSD and THC support the contention that the two drugs do not have the same basic mechanisms of action. Additionally, the overall pharmacological profiles of the two drugs are sufficiently diverse to suggest different substrates of action. As shown in the present study and in previous works (Consroe et al., 1975; Consroe et al., 1977; Pryor et al., 1976), depending upon the particular experimental procedure, THC and LSD can elicit both stimulant and depressant responses, and thus their combined effects are undoubtedly linked to these individual properties. However, the exact explanation of these interactive effects must await further experimentation.

\section{REFERENCES}

Agurell, A., Nilsson, I., Ohlsson, A., \& Sandberg, F. On the metabolism of tritium-labelled $\Delta^{9}$-tetrahydrocannabinol in the rabbit. Biochemical Pharmacology, 1979, 19, 1333-1339.

Consroe, P., Jones, B., \& Chin, L. Delta-9-tetrahydrocannabinol, EEG and behavior: The importance of adaptation to the testing milieu. Pharmacology, Biochemistry and Behavior, 1975, 3, 173-177.

Consroe, P., Jones, B., \& Martin, P. Lysergic acid diethylamide antagonism by chlorpromazine, haloperidol, diazepam and pentobarbital in the rabbit. Toxicology and Applied Pharmacology, 1977, 42, 45-54.

Dewey, W. L., Martin, B. R., \& Harris, L. S. Chronic effects of delta-9-tetrahydrocannabinol in animals: Tolerance and biochemical changes. In M. C. Braude \& S. Szara (Eds.), The pharmacology of marijuana. New York: Raven Press, 1976.

DuPont, R. Polydrug abuse and the maturing national drug abuse data base. Annals of the New York Academy of Science, 1976, 281, 311-320. 
Fujimori, M., \& Himwich, H. Delta-9-tetrahydrocannabinol and the sleep-wakefulness cycle in rabbits. Physiology \& Behavior, 1973, 11, 291-295.

ISBELL, H., \& JASINSKI, D. A comparison of LSD-25 with (-) delta-9-transtetrahydrocannabinol and attempted cross tolerance between LSD and THC. Psychopharmacologia, 1969, 14, 115-123.

Lipparini, F., Scotti de Carolis, A., \& Longo, V. G. A neuropharmacological investigation of some trans-tetrahydrocannabinol derivatives. Physiology \& Behavior, 1969, 4, 527532.

Martin, P., \& Consroe, P. Tolerance to delta-9-tetrahydrocannabinol in adapted and nonadapted rabbits. Pharmacology, Biochemistry and Behavior, 1978, 9, 753-758.

Pryor, G. T. Acute and subacute behavioral and pharmacological interactions of delta-9-tetrahydrocannabinol with other drugs.
In M. C. Braude \& S. Szara (Eds.), The pharmacology of marijuana. New York: Raven Press, 1976.

Pryor, G. T., Husain, S., Mitoma, C., \& Braude, M. Acute and subacute interactions between delta-9-tetrahydrocannabinol and other drugs in the rat. Annals of the New York Academy of Science, 1976, 281, 171-189.

Silva, M., Terassa, E., Carlini, E. A., Clausser, V., \& KORKE, F. Lack of cross-tolerance in rats among $(-)$ delta9-tetrahydrocannabinol (delta-9-THC), cannabis extract, mescaline and lysergic acid diethylamide (LSD-25). Psychopharmacologia, 1968, 13, 332-340.

(Received for publication April 7, 1980; review accepted May 18,1981 .) 